PROCEEDINGS OF THE

AMERICAN MATHEMATICAL SOCIETY

Volume 137, Number 10, October 2009, Pages 3291-3296

S 0002-9939(09)09936-5

Article electronically published on June 5, 2009

\title{
COHOMOLOGY OF LINE BUNDLES ON THE COTANGENT BUNDLE OF A GRASSMANNIAN
}

ERIC N. SOMMERS

(Communicated by Gail R. Letzter)

To Professor Shoji on the occasion of his 60th birthday

\begin{abstract}
We show that certain line bundles on the cotangent bundle of a Grassmannian arising from an anti-dominant character $\lambda$ have cohomology groups isomorphic to those of a line bundle on the cotangent bundle of the dual Grassmannian arising from the dominant character $w_{0}(\lambda)$, where $w_{0}$ is the longest element of the Weyl group of $S L_{l+1}(k)$.
\end{abstract}

\section{INTRODUCTION}

Let $k$ be an algebraically closed field of characteristic $p \geq 0$. Consider the algebraic group $G=S L_{l+1}(k)$. Let $T \subset B$ be a maximal torus contained in a Borel subgroup of $G$ and let $X^{*}(T)$ denote the characters of $T$. We choose positive roots and simple roots $\Pi$ in $X^{*}(T)$ that correspond to the Borel subgroup opposite to $B$. We index $\Pi=\left\{\alpha_{j}\right\}$ so that $\alpha_{1}$ is an extremal root and $\alpha_{j}$ is next to $\alpha_{j+1}$ in the Dynkin diagram of type $A_{l}$. Let $\left\{\omega_{i}\right\}$ be the fundamental weights of $G$ corresponding to $\Pi$. Let $\alpha^{\vee}$ be the coroot of the root $\alpha$ and let $\langle-,-\rangle$ denote the pairing of $X^{*}(T)$ and the cocharacters $X_{*}(T)$ of $T$.

For a rational representation $V$ of $B$, denote by $H^{*}(G / B, V)$, or just $H^{*}(V)$ when there is no ambiguity, the cohomology of the sheaf of sections of the vector bundle $G \times{ }^{B} V$ over $G / B$. For $\lambda \in X^{*}(T)$, we use the notation $\lambda$ both for a character of $T$ and for the one-dimensional representation of $T$ or $B$ that it defines.

Let $P$ denote the maximal proper parabolic subgroup containing $B$ corresponding to all the simple roots except $\alpha_{m}$. Thus $G / P$ identifies with the Grassmannian of $m$-planes in $(l+1)$-space. Let $\mathfrak{u}_{m}$ be the Lie agebra of the unipotent radical of $P$. Denote by $S^{n} \mathfrak{u}_{m}^{*}$ the $n$-th symmetric power of the linear dual of $\mathfrak{u}_{m}$.

The result of this paper is the following:

Theorem 1.1. Let $r$ be an integer in the range

$$
-|l+1-2 m|-1 \leq r \leq 0 .
$$

If $p=0$ or

$$
p>\max \{r, \min \{m, l+1-m\}\},
$$

Received by the editors June 17, 2008, and, in revised form, February 19, 2009.

2000 Mathematics Subject Classification. Primary 20G10; Secondary 14F05.

The author was supported in part by NSF grant DMS-0201826.

(C)2009 American Mathematical Society 
then there is a G-module isomorphism

$$
H^{i}\left(G / B, S^{n} \mathfrak{u}_{m}^{*} \otimes r \omega_{m}\right) \simeq H^{i}\left(G / B, S^{n+r m} \mathfrak{u}_{l+1-m}^{*} \otimes-r \omega_{l+1-m}\right) \text { for all } i, n \geq 0 \text {. }
$$

That the theorem is related to the cohomology of line bundles on the cotangent bundle of a Grassmannian goes as follows. First, since all roots have the same length, the cotangent bundle of $G / P$ identifies with the vector bundle $G \times{ }^{P} \mathfrak{u}_{m}$. Second, for $\lambda \in X^{*}(P)$, let $L_{\lambda}$ denote the corresponding line bundle on $G / P$. Let $\pi: G \times{ }^{P} \mathfrak{u}_{m} \rightarrow G / P$ be the natural map. The line bundle $L_{\lambda}$ can be pulled back to a line bundle $\pi^{*}\left(L_{\lambda}\right)$ on $G \times{ }^{P} \mathfrak{u}_{m}$. We have

$$
H^{i}\left(G \times^{P} \mathfrak{u}_{m}, \pi^{*}\left(L_{\lambda}\right)\right) \simeq \bigoplus_{n \geq 0} H^{i}\left(G / P, S^{n} \mathfrak{u}_{m}^{*} \otimes \lambda\right) .
$$

Finally,

$$
H^{i}(G / B, V) \simeq H^{i}(G / P, V) \text { for all } i \geq 0
$$

for any $P$-representation $V$. See Chapter 8 of Jantzen's notes [3] or [1] for a discussion of these facts, which hold for any parabolic subgroup of a semisimple group.

Theorem 1.1 was used in the papers 4 and 5] to prove that certain nilpotent varieties are normal 1 An analogous theorem for $G$ of type $D_{2 l+1}$ was proved and used in [5], although that theorem was stated only in characteristic zero. The usefulness of Theorem 1.1 lies in the fact that it can be used for arbitrary semisimple $G$ whenever $P$ is replaced by a parabolic subgroup of $G$ whose Levi subgroup $L$ contains a semisimple subgroup $M$ of type $A_{m-1} \times A_{l-m}$ such that $G$ contains a Levi subgroup $L^{\prime}$ of semisimple type $A_{l}$ where $M \subset L^{\prime}$ and $\left[L^{\prime}, L\right] \subset L$. Then multiple applications of Theorem 1.1 often lead to a situation, at least in characteristic zero, where the cohomology groups vanish for all $n \geq 0$ when $i>0$. The reason is that when $p=0$ we are in a position to invoke a version of the Grauert-Riemenschneider theorem.

Perhaps the most interesting feature of the theorem is that it translates the cohomology of a line bundle on the cotangent bundle of one partial flag variety into the cohomology of a line bundle on the cotangent bundle of a different partial flag variety.

\section{Preliminaries}

Recall the following proposition, due to Demazure and extended to positive characteristic by Thomsen. It is true for all semisimple groups, although we use it here only for type $A$. From now on, $P_{\alpha}$ refers to the minimal parabolic subgroup of $G$ containing $B$ corresponding to the simple root $\alpha$. If $\alpha=\alpha_{t}$, then we may write $P_{t}$ instead of $P_{\alpha_{t}}$.

Proposition 2.1 ([2], 6]). Let $V$ be a rational representation of $B$ and assume that $V$ extends to a representation of the parabolic subgroup $P_{\alpha}$. Let $\lambda \in X^{*}(T)$ be such that $s=\left\langle\lambda, \alpha^{\vee}\right\rangle \leq-1$. If $p=0$ or $p>-s$, then there is a G-module isomorphism

$$
H^{i}(G / B, V \otimes \lambda) \simeq H^{i-1}(G / B, V \otimes \lambda-(s+1) \alpha) \text { for all } i \geq 0 .
$$

In particular, if $s=-1$, then all cohomology groups $H^{i}(G / B, V \otimes \lambda)$ vanish.

This leads to the following result for $G=S L_{l+1}(k)$.

\footnotetext{
${ }^{1}$ The reference for the theorem in those papers is supplanted by the current paper.
} 
Lemma 2.2. Let $Q$ be a representation of $B$ that extends to a representation of each $P_{t}$ for $a \leq t \leq b$. Let $\lambda \in X^{*}(T)$ be such that $\left\langle\lambda, \alpha_{t}^{v}\right\rangle=0$ for $a<t \leq b$. Set $s=\left\langle\lambda, \alpha_{a}^{\vee}\right\rangle$ and assume that $a-b-1 \leq s \leq-1$. If $p=0$ or $p>-s$, then $H^{*}(Q \otimes \lambda)=0$.

Proof. This is an application of Proposition 2.1. utilizing it a total of $-s$ times, starting with the parabolic $P_{a}$. After one application we have

$$
H^{i}(Q \otimes \lambda) \simeq H^{i-1}\left(Q \otimes \lambda+(-s-1) \alpha_{a}\right) .
$$

After the second application we have that the latter is isomorphic to

$$
H^{i-2}\left(Q \otimes \lambda+(-s-1) \alpha_{a}+(-s-2) \alpha_{a+1}\right) .
$$

Continuing along, we find that after $-s-1$ times that

$$
H^{i}(Q \otimes \lambda) \simeq H^{i+s+1}\left(Q \otimes \lambda+(-s-1) \alpha_{a}+\cdots+2 \alpha_{a-s-3}+\alpha_{a-s-2}\right) .
$$

This is possible since $a-s-2<b$, and thus $Q$ extends to a representation of $P_{j}$ for $a \leq j \leq a-s-2<b$.

We are done at this point because

$$
\left\langle\lambda+(-s-1) \alpha_{a}+\cdots+\alpha_{a-s-2}, \alpha_{a-s-1}^{\vee}\right\rangle=-1
$$

and $a-s-1 \leq b$ so that $Q$ is a representation of $P_{a-s-1}$. Thus Proposition 2.1 applies again, giving the total vanishing of cohomology.

We can now prove the main result.

\section{Proof of Theorem 1.1}

Proof. By symmetry we may assume that $m \leq l+1-m$, so that the extremal value for $r$ is

$$
-|l+1-2 m|-1=2 m-l-2 .
$$

Step 1. In this step, $r$ may be an arbitrary integer. Consider the intersection $V=\mathfrak{u}_{m} \cap \mathfrak{u}_{l+1-m}$. We will show in Step 1 that for all $i, n$,

$$
H^{i}\left(S^{n} \mathfrak{u}_{m}^{*} \otimes r \omega_{m}\right) \simeq H^{i}\left(S^{n} V^{*} \otimes r \omega_{m}\right)
$$

We begin by taking the Koszul resolution of the short exact sequence

$$
0 \rightarrow U \rightarrow \mathfrak{u}_{m}^{*} \rightarrow V^{*} \rightarrow 0
$$

(this defines $U$ ) and tensoring it with $r \omega_{m}$. This gives

$$
0 \rightarrow \cdots \rightarrow S^{n-j} \mathfrak{u}_{m}^{*} \otimes \bigwedge^{j} U \otimes r \omega_{m} \rightarrow \cdots \rightarrow S^{n} \mathfrak{u}_{m}^{*} \otimes r \omega_{m} \rightarrow S^{n} V^{*} \otimes r \omega_{m} \rightarrow 0 .
$$

We claim that

$$
H^{*}\left(S^{n-j} \mathfrak{u}_{m}^{*} \otimes \bigwedge^{j} U \otimes r \omega_{m}\right)=0
$$

for $1 \leq j \leq \operatorname{dim} U$, from which Equation 1 will follow. The $T$-weights of $U$ are those of the form

$$
\alpha_{c}+\alpha_{c+1}+\cdots+\alpha_{d}
$$

where $c \leq m$ and $m \leq d<l+1-m$. Therefore, if $\lambda$ is a $T$-weight of $\bigwedge^{j} U$, there exists $a$ with $m<a \leq l+1-m$ such that $-m \leq\left\langle\lambda, \alpha_{a}^{\vee}\right\rangle \leq-1$ and $\left\langle\lambda, \alpha_{t}^{v}\right\rangle=0$ for $t>a$. 
Set $s=\left\langle\lambda, \alpha_{a}^{\vee}\right\rangle$ and $b=l$. We can invoke Lemma 2.2 for $\lambda$ and $Q:=S^{n-j} \mathfrak{u}_{m}^{*} \otimes$ $r \omega_{m}$. Indeed, $Q$ is stable under the parabolic subgroups $P_{t}$ for $t \geq a$. Also $a-b-1 \leq$ $-m$ since $a \leq l+1-m$ and so $s$ is in the range $a-b-1 \leq-m \leq s \leq-1$ and so the lemma applies, given our assumption on the characteristic of $k$. It follows that $H^{*}(Q \otimes \lambda)=0$ for all weights $\lambda$ appearing in $\bigwedge^{j} U$ for $1 \leq j \leq \operatorname{dim} U$. Thus if we filter $\Lambda^{j} U$ by $B$-subrepresentations such that the consecutive quotients are onedimensional, we deduce that $H^{*}\left(Q \otimes \bigwedge^{j} U\right)=0$ for $1 \leq j \leq \operatorname{dim} U$ and Equation 1 follows.

Step 2. Let $V_{1}=V \cap \mathfrak{u}_{m-1}$ and $V_{2}=V_{1} \cap \mathfrak{u}_{l+2-m}$. If $m=1$, then $\mathfrak{u}_{m-1}$ and $\mathfrak{u}_{l+2-m}$ are considered to be the zero vector space. Let $\mu$ be a weight of the form

$$
r \omega_{m}+r^{\prime} \omega_{l+1-m}
$$

and assume that $2 m-2-l \leq r \leq-1$ with $r^{\prime}$ unrestricted, unless $r=2 m-2-l$, in which case assume that $r^{\prime}=0$. In this step we show for all $n \geq 0$ that

$$
H^{*}\left(S^{n} V_{1}^{*} \otimes \mu\right)=0 \text {. }
$$

Take the Koszul resolution of

$$
0 \rightarrow U_{2} \rightarrow V_{1}^{*} \rightarrow V_{2}^{*} \rightarrow 0
$$

(this defines $U_{2}$ ) and tensor it with $\mu$. We will show that

$$
H^{*}\left(S^{n} V_{2}^{*} \otimes \mu\right)=0
$$

and

$$
H^{*}\left(S^{n-j} V_{1}^{*} \otimes \bigwedge^{j} U_{2} \otimes \mu\right)=0
$$

for $1 \leq j \leq m-1$, and then Equation 2 will follow (the dimension of $U_{2}$ is $m-1$ as shown below).

The subspace $V_{2}$ coincides with $\mathfrak{u}_{m-1} \cap \mathfrak{u}_{l+2-m}$. Hence $V_{2}^{*}$ is stable under $P_{t}$ for $m \leq t \leq l+1-m$. It follows that $H^{*}\left(S^{n} V_{2}^{*} \otimes \mu\right)=0$ by Lemma 2.2 with $a=m, b=l-m$ unless $r^{\prime}=0$, in which case $b=l+1-m$. In all cases, we have $a-b-1 \leq r \leq-1$ by hypothesis and the lemma applies since we are assuming $p>-r$.

Now the weights of $U_{2}$ are

$$
\alpha_{c}+\alpha_{c+1}+\cdots+\alpha_{l+1-m},
$$

where $1 \leq c \leq m-1$. If $\lambda$ is a weight of $\bigwedge^{j} U_{2}$, then $\lambda$ satisfies $\left\langle\lambda, \alpha_{l+2-m}^{\vee}\right\rangle=-j$ and $\left\langle\lambda, \alpha_{t}^{\vee}\right\rangle=0$ for $t>l+2-m$. Consequently, if we filter $\wedge^{j} U_{2}$ as in Step 1 and apply Lemma 2.2, we get

$$
H^{*}\left(S^{n-j} V_{1}^{*} \otimes \bigwedge^{j} U_{2} \otimes \mu\right)=0
$$

for $1 \leq j \leq m-1$. The lemma works with $a=l+2-m, b=l$. Thus $a-b-1=$ $(l+2-m)-l-1=-m+1$ and $j$ is in the acceptable range $-m+1 \leq-j \leq-1$. We are also using the fact that $S^{n-j} V_{1}^{*} \otimes \mu$ is stable under $P_{t}$ for $t \geq l+2-m$.

Step 3. In this step, we show that for all $i, n$,

$$
H^{i}\left(S^{n} V^{*} \otimes \mu\right) \simeq H^{i}\left(S^{n-m} V^{*} \otimes \mu+\omega_{m}+\omega_{l+1-m}\right)
$$

for $\mu$ as in Step 2, 
We take the Koszul resolution of the short exact sequence

$$
0 \rightarrow U_{1} \rightarrow V^{*} \rightarrow V_{1}^{*} \rightarrow 0
$$

(this defines $U_{1}$ ) and tensor it with $\mu$ to get

$$
\begin{aligned}
& 0 \rightarrow S^{n-m} V^{*} \otimes \bigwedge^{m} U_{1} \otimes \mu \rightarrow \cdots \rightarrow S^{n-j} V^{*} \otimes \bigwedge^{j} U_{1} \otimes \mu \rightarrow \ldots \\
& \rightarrow S^{n} V^{*} \otimes \mu \rightarrow S^{n} V_{1}^{*} \otimes \mu \rightarrow 0 .
\end{aligned}
$$

The weights of $U_{1}$ are of the form

$$
\alpha_{m}+\alpha_{m+1}+\cdots+\alpha_{d}
$$

where $l+1-m \leq d \leq l$ (and in particular, $\operatorname{dim} U_{1}=m$ ). We study the terms $\bigwedge^{j} U_{1}$ for $1 \leq j<m$. If $\lambda$ is a weight of $\bigwedge^{j} U_{1}$, then $\lambda$ satisfies $\left\langle\lambda, \alpha_{m-1}^{\vee}\right\rangle=-j$ and $\left\langle\lambda, \alpha_{t}^{\vee}\right\rangle=0$ for $t<m-1$. Consequently, proceeding as in Step 1, we filter $\wedge^{j} U_{1}$ and apply Lemma 2.2 (after applying an outer automorphism to $G$ to arrive at the obvious symmetric setup) to get

$$
H^{*}\left(S^{n-j} V^{*} \otimes \bigwedge^{j} U_{1} \otimes \mu\right)=0
$$

when $j<m$. The lemma works with $a=1, b=m-1$, so that $a-b-1=-m+1 \leq$ $-j \leq-1$. We note that $V$ is a representation of $P_{t}$ for $t \leq m-1$.

On the other hand, for the case $j=m$, we have

$$
\bigwedge^{m} U_{1}=m\left(\alpha_{m}+\alpha_{m+1}+\cdots+\alpha_{l+1-m}\right)+(m-1) \alpha_{l+2-m}+\cdots+2 \alpha_{l-1}+\alpha_{l} .
$$

So $m-1$ applications of Proposition Demazure as in the proof of Lemma 2.2 yield

$$
H^{i}\left(S^{n-m} V^{*} \otimes \bigwedge^{m} U_{1} \otimes \mu\right) \simeq H^{i+m-1}\left(S^{n-m} V^{*} \otimes \mu+\omega_{m}+\omega_{l+1-m}\right) .
$$

By breaking Equation 4 into short exact sequences and taking their cohomology, we conclude that

$$
H^{i}\left(S^{n} V^{*} \otimes \mu\right) \simeq H^{i}\left(S^{n-m} V^{*} \otimes \mu+\omega_{m}+\omega_{l+1-m}\right),
$$

where we are using

$$
H^{*}\left(S^{n} V_{1}^{*} \otimes \mu\right)=0
$$

from Step 2 .

Step 4 . We obtain the theorem by using Step 3 repeatedly, starting with $\mu=r \omega_{m}$ with $r$ in the prescribed range of the statement of the theorem. After $-r$ steps we arrive at

$$
H^{i}\left(S^{n} V^{*} \otimes r \omega_{m}\right) \simeq H^{i}\left(S^{n+r m} V^{*} \otimes-r \omega_{l+1-m}\right),
$$

for all $i, n$.

The proof is completed by using Step 1 and the symmetric version of Equation 1 which gives

$$
H^{i}\left(S^{n+r m} V^{*} \otimes-r \omega_{l+1-m}\right) \simeq H^{i}\left(S^{n+r m} \mathfrak{u}_{l+1-m}^{*} \otimes-r \omega_{l+1-m}\right)
$$

for all $i, n$. 


\section{REFERENCES}

[1] A. Broer, Normality of Some Nilpotent Varieties and Cohomology of Line Bundles on the Cotangent Bundle of the Flag Variety, Lie Theory and Geometry, Progr. Math., 123, Birkhäuser, Boston, 1994, pp. 1-19. MR1327529 (96g:14042)

[2] M. Demazure, A very simple proof of Bott's Theorem, Invent. Math. 33 (1976), 271-272. MR0414569 (54:2670)

[3] J. C. Jantzen, Nilpotent orbits in representation theory, Lie theory, Progr. Math., 228, Birkhäuser, Boston, 2004, pp. 1-211. MR2042689 (2005c:14055)

[4] E. Sommers, Normality of nilpotent varieties in $E_{6}$, J. Algebra 270 (2003), no. 1, 288-306. MR2016663 (2004i:20085)

[5] , Normality of very even nilpotent varieties in $D_{2 l}$, Bull. London Math. Soc. 37 (2005), no. 3, 351-360. MR 2131388 (2005k:20116)

[6] J. F. Thomsen, Normality of certain nilpotent varieties in positive characteristic, J. Algebra 227 (2000), 595-613. MR.1759837 (2001g:14079)

University of Massachusetts-Amherst, Amherst, Massachusetts 01003

E-mail address: esommers@math . umass .edu 